\title{
Caracterização físico-química de méis produzidos no Estado do Piauí para diferentes floradas ${ }^{1}$
}

\author{
Claudécia L. da Silva ${ }^{2}$, Alexandre J. de M. Queiroz ${ }^{3}$ \& Rossana M. F. de Figueirêdo ${ }^{3}$
}

\footnotetext{
1 Parte da Dissertação de Mestrado do primeiro autor, apresentada na UFPB

2 Fone: (86) 422-1902. E-mail: claudecia@yahoo.com (Foto)

${ }^{3}$ DEAg/CCT/UFCG, Av. Aprígio Veloso 882, CP 10.017, CEP 58109-970. Campina Grande, PB. Fone: (83) 310-1287. E-mail: alex@deag.ufcg.edu.br, rossana@deag.ufcg.edu.br
}

Protocolo 174 - 21/11/2002 - Aprovado em 7/7/2004

\begin{abstract}
Resumo: Este trabalho foi realizado com o objetivo de se caracterizar físico-químicamente méis de Apis mellifera L. produzidos no Estado do Piauí, a partir de floradas típicas da região semi-árida do Nordeste brasileiro e, ao mesmo tempo, avaliar a qualidade desses produtos. Analisaram-se os méis produzidos a partir das floradas de marmeleiro (Croton sonderianus Müll. Arg.), jitirana (Hyptis suaveolens), camaratuba (Cratylia mollis Mart. ex Benth), silvestre (Serjania glabrata) e angico de bezerro (Piptadenia moniliformis), seguindo-se as metodologias recomendadas pelo Ministério da Agricultura e do Abastecimento, através de Instrução Normativa de outubro/ 2000. Foram realizadas as seguintes análises: umidade, hidroximetilfurfural (HMF), açúcares redutores, sacarose aparente, cinzas, acidez livre, sólidos insolúveis em água, atividade diastásica, ${ }^{\circ}$ Brix e pH. Os valores individuais de cada variável analisada, comparados com os padrões estabelecidos pelo Ministério da Agricultura e do Abastecimento, apresentaram resultados satisfatórios encontrando-se todos os méis analisados, dentro dos padrões exigidos na citada Instrução Normativa.
\end{abstract}

Palavras-chave: Apis mellifera L., mel floral, controle de qualidade

\section{Physical and chemical characterization of honeys produced in the State of Piauí - Brazil}

\begin{abstract}
The objective of this work was to evaluate the physical-chemical composition of honeys produced in the Piauí State, located in semi-arid region of Northeast Brazil. The honeys produced from 'marmeleiro' (Croton sonderianus Müll. Arg.), 'jitirana' (Hyptis suaveolens), 'camaratuba' (Cratylia mollis Mart. ex Benth), 'silvestre' (Serjania glabrata) and 'angico de bezerro' (Piptadenia moniliformis), were analysed following the methods recommended by the Brazilian Ministry of Agriculture and Normative Instruction of October 2000. The following analyses were performed: moisture, hydroxymethylfurfural (HMF), reducing sugars, apparent sucrose, ash, free acidity, insoluble solids in water, diastatic activity, ${ }^{\circ}$ Brix and $\mathrm{pH}$. The individual values of each analyzed parameter were compared with the standards established by Ministry of Agriculture and Suplies and presented satisfactory results, matching the standards cited by the Normative Instruction.
\end{abstract}

Key words: Apis mellifera L., floral honey, quality control

\section{INTRODUÇÃO}

O homem tem utilizado o mel de diversas maneiras, seja como alimento, ou como medicamento, devido às suas propriedades anti-sépticas e, ainda, como conservante de frutas e grãos (Cortopassi-Laurino \& Gelli, 1991).

Entende-se que mel é um produto da abelha, a única capaz de produzi-lo. Esta observação é importante, haja vista a elaboração artificial de certos produtos, como a glicose de milho, comercializados como mel, ou utilizados para sua adulteração. Assim, pode-se definir o mel como o produto alimentício produzido pelas abelhas melíferas, a partir do néctar das flores ou, também, das secreções procedentes de partes vivas das plantas ou de excreções de insetos sugadores de plantas que ficam sobre partes vivas de plantas, que as abelhas recolhem, transformam, combinam com substâncias específicas próprias, 
armazenam e deixam madurar nos favos da colméia (Brasil, 2000).

O mel puro deve apresentar aspecto líquido, denso, viscoso e translúcido, e cor que poderá variar do amarelo ao amareloavermelhado, com cheiro próprio, sabor doce e característico (Catalan, 1981).

O mel não deve ser caracterizado diretamente como de origem animal, já que a abelha não o secreta (Campos, 1987), mas o elabora a partir de matérias-primas coletadas em campo. Sendo assim processado, o produto final está sujeito à sua origem, com características dependendo da concentração, nas matérias-primas, de açúcares, minerais e vitaminas, entre outros, que serão a base para a composição final do mel. Sendo este fato amplamente reconhecido, é corriqueiro afirmar-se que o aroma, sabor e a cor do produto, são variáveis em função da sua origem floral (Crane, 1983) e, para fins de comercialização, o mel pode ser classificado de acordo com sua origem botânica e procedimento de obtenção (Brasil, 2000).

Quando se trabalha com mel, é comum encontrar variações na sua composição física e química, tendo em vista que variados fatores interferem na sua qualidade, como condições climáticas, estádio de maturação, espécie de abelha, processamento e armazenamento, além do tipo de florada.

Este trabalho foi realizado com o objetivo de se caracterizar, físico-quimicamente, e avaliar a qualidade dos méis de Apis mellifera L. produzidos no Estado do Piauí, selecionados desde sua origem floral, proveniente de cinco espécies botânicas nativas: marmeleiro (Croton sonderianus Müll. Arg.), jitirana (Hyptis suaveolens), camaratuba (Cratylia mollis Mart. ex Benth), silvestre (Serjania glabrata) e angico de bezerro (Piptadenia moniliformis).

\section{MATERIAL E MÉTODOS}

Os méis utilizados nos ensaios foram produzidos no Estado do Piauí, nos municípios de Picos, Itainópolis, Vera Mendes e Isaías Coelho, elaborados predominantemente das floradas de marmeleiro (Croton sonderianus Müll. Arg.), silvestre (Serjania glabrata), angico de bezerro (Piptadenia moniliformis), jitirana (Hyptis suaveolens) e camaratuba (Cratylia mollis Mart. ex Benth), e coletados no decorrer do ano de 2000, nos meses de janeiro (marmeleiro e silvestre), em fevereiro, abril e julho, respectivamente. Para as análises físico-químicas utilizaram-se as metodologias recomendadas pelo Ministério da Agricultura e do Abastecimento através, de Instrução Normativa de outubro/2000.

\section{Obtenção das amostras}

As amostras foram obtidas a partir de coletas realizadas por apicultores, associações e cooperativas de apicultura, no decorrer do ano 2000; em seguida, foram encaminhadas ao Laboratório de Armazenamento e Processamento de Produtos Agrícolas, na Universidade Federal de Campina Grande e em cada amostra foi feita a identificação individual quanto à origem botânica e identificação da localidade onde foi coletada, de acordo com as informações obtidas junto aos fornecedores; depois, foram centrifugadas, filtradas, decantadas, acondicionadas em recipientes apropriados e armazenadas para a utilização nos ensaios.

\section{Avaliação das características físico-químicas}

Umidade: A umidade dos méis foi determinada por refratometria, segundo o Método $n^{\circ} 969.38$ b (AOAC, 1997) recomendado como metodologia oficial do Ministério da Agricultura e do Abastecimento (Brasil, 2000). O princípio deste método consiste na determinação do índice de refração do mel a $20^{\circ} \mathrm{C}$, que é convertido para o conteúdo de umidade através de uma tabela de referência a qual, por sua vez, fornece a concentração como uma função do índice de refração.

Hidroximetilfurfural (HMF): Foi determinado através do método quantitativo, que consiste na verificação do $\mathrm{HMF}$, utilizando-se método espectrofotométrico a 284 e $336 \mathrm{~nm}$, conforme o método $n^{\circ} 980.23$ (AOAC, 1997) recomendado pela Instrução Normativa do Ministério da Agricultura e do Abastecimento (Brasil, 2000).

Açúcares redutores: Foram determinados segundo o método do CAC (1990) a partir da modificação do procedimento de Lane e Eynon, envolvendo a redução da solução de Fehling, modificada por Soxhlet, durante a titulação no ponto de ebulição com uma solução de açúcares redutores do mel, usando-se azul de metileno como indicador.

Sacarose aparente: A sacarose aparente foi mensurada de acordo com o método do item 7.2 do CAC (1990) o qual determina a sacarose aparente após a inversão por hidrólise ácida, conforme recomendado pela Instrução Normativa do Ministério da Agricultura e do Abastecimento (Brasil, 2000).

Cinzas: Da incineração das amostras em mufla aquecida a $600^{\circ} \mathrm{C}$, determinou-se a quantidade de cinzas nos méis (CAC, 1990), conforme recomendado pelo Ministério da Agricultura e do Abastecimento (Brasil, 2000) por meio do qual é possível se determinar algumas irregularidades no mel, como, por exemplo, contaminação provocada pela não decantação e/ou filtração no final do processo de extração do mel.

Acidez livre: A acidez livre foi determinada de acordo com o método no 962.19 da AOAC (1997), que se baseia na titulação da amostra, com solução de $\mathrm{NaOH} 0,05 \mathrm{~N}$, até atingir o $\mathrm{pH} 8,5$, cujo método é recomendado pelo Regulamento Técnico de Identidade e Qualidade do Mel (Brasil, 2000).

Sólidos insolúveis em água: Segundo o método CAC (1990), determinou-se o teor de sólidos insolúveis em mel, por gravimetria, de acordo com a recomendação do Ministério da Agricultura e do Abastecimento (Brasil, 2000).

Atividade diastásica: Foi determinada pelo método CAC (1990), cujo resultado foi expresso em $\mathrm{mL}$ de solução de amido a $1 \%$ hidrolisado pela enzima em $1 \mathrm{~g}$ de mel, em $1 \mathrm{~h}$. Este método é recomendado também pelo Ministério da Agricultura e do Abastecimento (Brasil, 2000).

Análise estatística: A comparação entre as médias dos dados experimentais das análises físico-químicas foi feita pelo teste de Tukey, utilizando-se o programa computacional Assistat 6.2 (Silva, 1996). 


\section{RESULTADOS E DISCUSSÃO}

Na Tabela 1 são apresentados os valores médios de três repetições das análises físico-químicas dos méis obtidos a partir de diferentes floradas.

Tabela 1. Características físico-químicas de méis produzidos com as floradas de marmeleiro (MM), jitirana (JT), camaratuba $(\mathrm{CM})$, silvestre (SV) e angico de bezerro $(\mathrm{AB})^{*}$

\begin{tabular}{|c|c|c|c|c|c|}
\hline \multirow{2}{*}{ Características } & \multicolumn{5}{|c|}{ Floradas } \\
\hline & MM & JT & $\mathrm{CM}$ & SV & $\mathrm{AB}$ \\
\hline Umidade (\%) & $18,8 \mathrm{~d}$ & $19,5 \mathrm{c}$ & $17,6 \mathrm{e}$ & $19,6 b$ & $19,7 \mathrm{a}$ \\
\hline $\mathrm{HMF}^{* *}\left(\mathrm{mg} \mathrm{kg}^{-1}\right)$ & $0,30 \mathrm{e}$ & $8,96 \mathrm{a}$ & $1,45 \mathrm{~d}$ & $4,33 b$ & $3,44 \mathrm{c}$ \\
\hline Açúcares Redutores (\%) & $68,92 \mathrm{e}$ & $71,57 \mathrm{~d}$ & $83,59 b$ & $85,49 \mathrm{a}$ & $76,36 \mathrm{c}$ \\
\hline Sacarose Aparente (\%) & $2,93 b$ & $3,07 \mathrm{a}$ & $2,55 \mathrm{c}$ & $1,57 \mathrm{e}$ & $2,24 \mathrm{~d}$ \\
\hline Cinzas $(\%)$ & $0,07 \mathrm{c}$ & $0,06 \mathrm{c}$ & $0,10 \mathrm{~b}$ & $0,11 b$ & $0,14 \mathrm{a}$ \\
\hline Acidez Livre (meq kg${ }^{-1}$ ) & $25,43 \mathrm{c}$ & $10,10 \mathrm{e}$ & $28,33 b$ & $31,03 a$ & $19,99 d$ \\
\hline SAI** $(\%)$ & $0,08 b$ & $0,06 \mathrm{c}$ & $0,09 a$ & $0,08 b$ & $0,08 b$ \\
\hline Atividade Diastásica (DN) & $10,11 \mathrm{c}$ & $10,08 \mathrm{c}$ & $20,83 a$ & $20,74 a$ & $17,65 b$ \\
\hline${ }^{\circ}$ Brix & $76,07 \mathrm{e}$ & $79,20 \mathrm{c}$ & $80,80 \mathrm{a}$ & $79,50 \mathrm{~b}$ & $78,70 \mathrm{~d}$ \\
\hline $\mathrm{pH}$ & $3,54 \mathrm{e}$ & $3,70 \mathrm{~d}$ & $3,75 \mathrm{c}$ & $4,68 b$ & $5,30 \mathrm{a}$ \\
\hline
\end{tabular}

De acordo com a análise estatística das características físico-químicas das amostras de méis analisadas (Tabela 1) as variáveis: umidade, hidroximetilfurfural, açúcares redutores, sacarose aparente, acidez livre, ${ }^{\circ}$ Brix e $\mathrm{pH}$, apresentaram diferenças significativas pelo teste de Tukey a $5 \%$ de probabilidade entre todas as amostras estudadas, denotando-se a influência que as espécies florais tiveram sobre o nível desses componentes. Os teores de minerais (cinzas), sólidos insolúveis em água e atividade diastásica, foram menos dependentes da origem floral dos méis, como demonstram alguns valores para essas características que não apresentaram diferenças significativas.

\section{Umidade}

O mel de abelha piauiense apresentou teor médio de umidade de 19,4\%, para um intervalo de variação de 17,6 a 19,7\% (Tabela 1). Esses valores se encontram abaixo do limite máximo permitido pela legislação vigente, de $20 \%$, estabelecido pelo Ministério da Agricultura e do Abastecimento (Brasil, 2000), estando próximos dos encontrados por diversos autores, que relatam teores médios respectivos de 19\% (Ramalho et al.,1987), 19,8\% (Campos, 1987), 21\% (Duran et al., 1996) e 17,8\% (Noronha, 1997) e que trabalharam com méis da região semi-árida, de clima semelhante ao da região em que as amostras do presente estudo foram produzidas.

Apenas uma amostra não apresentou teor superior a 18\% mas, dentre as amostras com percentuais de umidade maior que $18 \%$, três foram coletadas nos meses de janeiro a abril, período em que se concentram as chuvas na região de coleta das amostras. É provável que os méis produzidos durante a época chuvosa apresentem maior umidade em função da saturação do ar e do grande fluxo de néctar que ocorre logo após as chuvas, o que pode constituir dificuldade às abelhas para remoção da água, como afirmam Ramalho (1985) e Noronha (1997); isto confirma a observação dos apicultores de que os méis produzidos no primeiro semestre do ano são menos viscosos, podendo-se atribuir a isto a menor concentração do néctar, que se apresenta mais aquoso. Assim, as chuvas e a saturação do ar pela água provocariam a diluição do néctar. Esses valores podem ainda estar relacionados às falhas na manipulação, podendo trazer problemas na conservação, uma vez que todo mel com umidade superior a $18 \%$ está sujeito a fermentação (Gonnet, 1982; Noronha, 1997).

\section{Hidroximetilfurfural (HMF)}

O teor médio de HMF no mel estudado no presente trabalho (Tabela 1) foi de 3,7 $\mathrm{mg} \mathrm{kg}^{-1} \mathrm{de}$ mel, para um intervalo de $0,30 \mathrm{a}$ $8,96 \mathrm{mg} \mathrm{kg}^{-1}$. Os valores se encontram dentro dos padrões exigidos pela legislação brasileira vigente, uma vez que o Ministério da Agricultura e do Abastecimento estabelece um máximo de $40 \mathrm{mg} \mathrm{kg}^{-1}$ (Brasil, 2000). O mel da florada de jitirana apresentou mais elevado teor de $\operatorname{HMF}\left(8,96 \mathrm{mg} \mathrm{kg}^{-1}\right)$, seguido do mel de silvestre $\left(4,33 \mathrm{mg} \mathrm{kg}^{-1}\right)$. No mel da florada de marmeleiro observou-se o menor teor, que foi de $0,30 \mathrm{mg} \mathrm{kg}^{-1}$. Os valores para as amostras estudadas do presente trabalho se encontram próximos ao determinado por Bianchi (1989). Constatou-se, ainda, que os méis mais escuros (camaratuba e silvestre) nem sempre apresentaram valores maiores de HMF em relação aos méis mais claros (marmeleiro e jitirana), divergindo do que foi observado por Issa et al. (1998) ao avaliarem méis de apis, jataí e melipona provenientes do Brasil, Chile e Estados Unidos, mas o autor não menciona a origem floral das amostras.

O HMF é utilizado como indicador de qualidade, uma vez que tem origem na degradação de enzimas presentes nos méis e apenas uma pequena quantidade de enzima é encontrada em méis maduros. Teoricamente, méis com maior taxa de frutose darão origem a maiores taxas de HMF, ao longo de processos de armazenagem. Pequenas quantidades de HMF são encontradas em méis recém-colhidos, mas valores mais significativos podem indicar alterações importantes provocadas por armazenamento prolongado em temperatura ambiente alta e/ou superaquecimento (Vilhena \& AlmeidaMuradian, 1999a) ou adulterações provocadas por adição de açúcar invertido. Embora alguns autores reportem aumentos no teor de HMF com o tempo de estocagem (Duran et al., 1996) tais acréscimos seriam em pequena proporção, como observado em Faria (1993) que relata aumento de HMF em méis armazenados durante $450 \mathrm{~d}$, com valor máximo de $3,5 \mathrm{mg} \mathrm{kg}^{-1}$.

\section{Açúcares redutores}

Os cinco tipos de méis apresentaram teor médio de açúcares redutores de 77,25\%, com valores mínimo e máximo de 68,92 e $85,49 \%$, respectivamente (Tabela 1). Esses valores estão próximos aos determinados por Komatsu \& Marchini (1996b) trabalhando com amostras de méis de flores silvestres de diferentes municípios de São Paulo, sem citar a época de coleta e Sodré et al. (2001) com méis do litoral norte da Bahia, omitindo a origem floral e a época de coleta. Tais teores se enquadram 
nos padrões estabelecidos pelo Ministério da Agricultura e do Abastecimento (Brasil, 2000) que exige um mínimo de açúcares redutores de $65 \%$, para méis de flores.

Os méis com coloração mais escura (silvestre e de camaratuba) apresentaram os maiores percentuais de açúcares redutores $(85,49$ e $83,59 \%$, respectivamente). De acordo com Cortopassi-Laurino \& Gelli (1991) a cor mais escura é uma característica dos méis que contêm maiores quantidades de açúcares redutores.

\section{Sacarose aparente}

O percentual de sacarose aparente apresentou valor médio igual a 2,5\%, com intervalo de variação de 1,57 a 3,07\% (Tabela 1). A amostra indicou maior percentual foi a da florada de jitirana, com $3,07 \%$, seguida do mel de marmeleiro, com $2,93 \%$, e o que apresentou menor percentual foi o de silvestre, com apenas $1,57 \%$. Komatsu \& Marchini (1996b) analisando méis de flores silvestres produzidos por Apis mellifera no Estado de São Paulo, encontraram uma faixa de variação de 0,2 a 2,74\% e valor médio de $2,4 \%$ de sacarose aparente. Percentuais semelhantes foram encontrados por Bonilla (2000) em méis de Mato Grosso do Sul, Cortopassi-Laurino \& Gelli (1991) e Noronha (1997), este último trabalhando com méis do Ceará e identificando 27 espécies vegetais na composição das amostras.

De acordo com os dados da Tabela 1, verifica-se que as amostras analisadas se encontram dentro dos padrões técnicos de identidade e qualidade de mel, estabelecidos pelo Ministério da Agricultura e do Abastecimento (Brasil, 2000), sendo o percentual máximo de sacarose aparente permitido de $6 \%$ para méis de flores e de $15 \%$ para méis de melato; no entanto, Sodré et al. (2001) afirmaram que a proporção de sacarose no mel deve ser em torno de $2-3 \%$ e, quando esse valor é muito alto, torna-se um indicativo do produto tratar-se de mel verde ou adulterado.

\section{Cinzas}

De acordo com os resultados das análises estatísticas, as médias obtidas para os teores de cinzas diferiram estatisticamente pelo teste de Tukey, entre os grupos: marmeleiro/ jitirana, camaratuba/silvestre e angico de bezerro (Tabela 1), mas se apresentaram estatisticamente iguais nos méis originados de marmeleiro/jitirana e camaratuba/silvestre. $\mathrm{O}$ percentual de cinzas médio foi de $0,10 \%$ para um intervalo de variação de 0,06 a $0,14 \%$, como se vê na Tabela 1 , cujos valores são próximos aos determinados por vários autores como Carvalho et al. (1998), trabalhando com méis de seis municípios baianos, e Marchini \& Moreti (2001), em méis provenientes de cinco espécies de eucalipto, e se encontram dentro dos padrões estabelecidos pela legislação brasileira para méis de abelha, que admite um valor máximo de $0,6 \%$ de cinzas (Brasil, 2000).

Observou-se, ainda, diferença considerável nos teores de cinza dos méis de coloração mais clara (marmeleiro e jitirana) em relação aos demais, com o mel de jitirana, que apresentou percentual de cinzas inferior aos demais $(0,06 \%)$, seguido do mel de marmeleiro $(0,07 \%)$. Esta correspondência entre cor e teor de minerais está de acordo com o relatado por FellerDemalsy et al. (1989) que, ao analisarem méis do Canadá, constataram que os de cor clara apresentam menor quantidade desses constituintes. Também Crane (1983) afirma que os méis com coloração mais escura, variando do âmbar ao âmbar escuro, tendem a apresentar maiores quantidades de minerais, embora não se apresente uma explicação para a causa.

\section{Acidez livre}

A acidez livre do mel expressa em termos de meq $\mathrm{kg}^{-1}$, variou de 10,10 a 31,03 (Tabela 1), mas esses valores estão dentro dos padrões de qualidade recomendados pela legislação, que determina um limite máximo de $60 \mathrm{meq} \mathrm{kg}^{-1}$ de mel (Brasil, 2000). Resultados semelhantes foram determinados por Noronha (1997) e Bonilla (2000). Cortopassi-Laurino \& Gelli (1991) determinaram uma média de acidez livre para méis de $12,1 \mathrm{meq} \mathrm{kg}^{-1}$ de mel, enquanto Faria (1993) obteve, para o mel de abelha elaborado a partir da florada de citrus, um valor médio de 9,0 meq $\mathrm{kg}^{-1}$ de mel.

A grande variação encontrada neste trababalho confirma as afirmações de Gonnet (1982) que relata, para acidez em méis de abelha, um intervalo de 10 a 60 meq $\mathrm{kg}^{-1}$ de mel. Os tipos de florada explicariam essa variabilidade, uma vez que a acidez no mel tem origem nos diversos ácidos orgânicos contidos no néctar coletado pelas abelhas (Root, 1985) que, pela ação da glicose-oxidase, originam o ácido glucônico (White Júnior, 1989) sendo ainda influenciada pela quantidade de minerais presentes no néctar (Barth, 1989).

\section{Sólidos insolúveis em água}

Os resultados das análises estatísticas mostram que os teores de sólidos insolúveis dos méis de marmeleiro, silvestre e angico de bezerro, não diferiram estatisticamente entre si, compondo um grupo com teores idênticos. Nos méis de jitirana e camaratuba os valores diferem entre si e em relação aos demais. $\mathrm{O}$ valor médio percentual de sólidos insolúveis em água nos méis analisados foi de $0,08 \%$ com uma variação de 0,06 a $0,09 \%$ (Tabela 1). Valores próximos a esses foram determinados por Vilhena \& Almeida-Muradian (1999b). As amostras analisadas encontram-se dentro dos padrões estabelecidos pela legislação, que estabelece um teor máximo de sólidos insolúveis em água de $0,1 \%$, tanto para o mel de flores como para o de melato (Brasil, 2000).

\section{Atividade diastásica (DN)}

Os resultados da análise estatística indicam que, assim como observado nos teores de cinzas e sólidos insolúveis, a diferença de floradas nem sempre influenciou os valores da atividade diastásica, de maneira que as amostras das floradas de camaratuba e silvestre não apresentaram diferenças significativas entre si pelo teste de Tukey, a nível de 5\% de probabilidade, o mesmo ocorrendo com os méis das floradas de marmeleiro e jitirana.

O índice médio de diástase nos méis analisados foi de 15,9 $\pm 5,42 \mathrm{DN}$, com os valores variando de 10,08 a $20,83 \mathrm{DN}$, apresentado na Tabela 1. Valores idênticos foram determinados por Komatsu \& Marchini (1996a) e Rodrigues et al. (1996), este trabalhando com méis resultantes de abelhas alimentadas artificialmente, com xarope de açúcar. Comparando-se os resultados obtidos com os estabelecidos pela legislação brasileira para qualidade de mel, constata-se que as amostras analisadas estão em conformidade com as especificações 
normatizadas, que estabelecem que o mínimo de atividade diastásica no mel deve ser de $8 \mathrm{DN}$ (escala Göethe).

Verificaram-se tons mais escuros nos méis com os maiores índices de diástase, (camaratuba e silvestre), com valores médios iguais a 20,83 e 20,74 DN, respectivamente, confirmando o observado por Bianchi (1989) ao estudarem méis da Argentina, e Feller-Demalsy et al. (1989) ao analisarem méis do Canadá. Campos (1987) também afirma que méis com maiores índices de diástase tendem a apresentar coloração escura.

\section{${ }^{\circ}$ Brix}

$\mathrm{O}^{\circ}$ Brix determinado nas amostras apresentou valor médio de $78,70^{\circ}$ Brix, em uma faixa de variação de 76,07 a $80,80^{\circ}$ Brix (Tabela 1) com o maior valor encontrado no mel da florada de camaratuba e o menor no mel da florada de marmeleiro. Tais valores são próximos aos encontrados por Carvalho et al. (1998) e Marchini \& Moreti (2001).

\section{pH}

Embora o pH não seja indicado, atualmente, como análise obrigatória no controle de qualidade dos méis brasileiros, mostra-se útil como variável auxiliar para avaliação da qualidade.

$\mathrm{O}$ valor médio do $\mathrm{pH}$ dos méis estudados foi de 4,2, variando entre 3,54 a 5,30 (Tabela 1). A média observada encontra-se próxima dos valores descritos por Ramalho (1985), Noronha (1997) e Duran et al. (1996) que verificaram valores de 3,95, 4,0 e 4,1 , respectivamente. Comparados aos resultados obtidos para méis produzidos em alguns Estados nordestinos, apresentados em Ramalho et al. (1987) os méis piauienses analisados neste trabalho apresentaram-se com valores de $\mathrm{pH}$ superiores. Variações observadas no pH, segundo Crane (1983) se devem, provavelmente, a particularidades da composição florística nas áreas de coleta, uma vez que o $\mathrm{pH}$ do mel pode ser influenciado pelo $\mathrm{pH}$ do néctar. Diferenças na composição do solo, afirma Noronha (1997), ou a associação de espécies vegetais para composição final do mel, podem também influenciar o $\mathrm{pH}$ deste produto.

\section{CONCLUSÕES}

1. Todos os méis estudados apresentaram características físico-químicas compatíveis com os valores referenciados na literatura e se enquadram dentro dos padrões de qualidade exigidos pelo Ministério da Agricultura, para a comercialização, no âmbito do mercado brasileiro.

2. Na maioria dos casos, o tipo de florada influenciou significativamente nas características físico-químicas dos méis estudados.

3. Os méis de coloração mais escura (camaratuba e silvestre) apresentaram valores maiores de açúcares redutores e maiores índices de atividade diastásica em relação aos méis mais claros. Os menores valores das cinzas foram observados nos méis mais claros (jitirana e marmeleiro).

\section{LITERATURA CITADA}

AOAC - Association of Official Analytical Chemists. Official methods of analysis. Washington, 1997, 1170p.
Barth, O.M. O pólen no mel brasileiro.1.ed. Rio de Janeiro: Gráfica Luxor, 1989. 151p.

Bianchi, E.M. Determinacion de HMF en la miel. Santiago del Estero: Centro de Investigaciones Apícolas/Universitad Nacional de Santiago del Estero, 1989. 81p.

Bonilla, L. Análises físico-químicas dos méis comercializados no Estado de Mato Grosso do Sul. In: Congresso Brasileiro de Apicultura, 13, 2000, Florianópolis. Resumos... Florianópolis: UFSC, 2000. CD Rom

Brasil. Instrução Normativa ${ }^{\circ} 11$, de 20 de outubro de 2000. Estabelece o regulamento técnico de identidade e qualidade do mel. Diário Oficial da República Federativa do Brasil, Brasília, 23 out. 2000. Seção 1, p.16-17.

CAC-Codex Alimentarius Commission. Official methods of analysis. v.3, Supl.2, 1990. p.15-39.

Campos, M.G.R. Contribuição para o estudo do mel, pólen, geleia real e própolis. Boletim da Faculdade de Farmácia de Coimbra, Coimbra, v.11, n.2, p.17-47, 1987.

Carvalho, C.A.L.; Marchini, L.C.; Teixeira, G.M.; Oliveira, P.C.F.; Rubia, V.R. Características físico-químicas de amostras de méis da Bahia. In: Congresso Brasileiro de Apicultura, 12, 1998, Salvador. Resumos... Salvador: FAABA, 1998. p.200.

Catalan, J.M.B. Relatório de atividades. Secretaria de Agricultura e Abastecimento do Piauí. Teresina, 1981. 27p.

Cortopassi-Laurino, M; Gelli, D.S. Analyse pollinique, propriétes physico-chimiques et action antibactérienne des miels d'abeilles africanisées Apis mellifera et de Méliponinés du Brésil. Apidologie, Paris, v.22, n.1, p.61-73, 1991.

Crane, E. O livro do mel. São Paulo: Nobel, 1983. 226p.

Durán, J.E.T.; Cortopassi-Laurino, M.; Issa, M.R.C.; Toledo, V.A.A.; Bastos, E.; Soares, A.E.E. Méis brasileiros: resultados de análises físico-químicas e palinológicas. In: Congresso Brasileiro de Apicultura, 11, Teresina, 1996. Resumos... Teresina: CBA, 1996. p 403-429.

Faria, J.A.F. Shelf life testing of honey. Ciência e Tecnologia de Alimentos, Campinas, v.13, n.1, p.58-66, 1993.

Feller-Demalsy, M.J.; Vicente, B.; Beaulieu, F. Teneur en mineraux et origine geographique des miels du Canada. Apidology, Paris, v.20, n.1, p.77-91, 1989.

Gonnet, M. Le miel: composition, proprietés, conservation. 2. ed. Montfavet: OPIDA, 1982. 109p.

Issa, M.R.C.; De Jong, D.; Horn, H. Determinação físico-química de amostras de méis normais, aquecidos e misturados. In: Congresso Brasileiro de Apicultura, 12, 1998, Salvador. Resumos... Salvador: FAABA, 1998. p.209.

Komatsu, S.S.; Marchini, L.C. Determinação do índice de diastase e hidroximetilfurfural de amostras de méis de flores silvestres produzidos por Apis mellifera no Estado de São Paulo. In: Congresso Brasileiro de Apicultura, 11, 1996, Teresina. Resumos... Teresina: CBA, 1996a. 343.

Komatsu, S.S.; Marchini, L.C. Teores de açúcares redutores e sacarose de amostras de méis de flores silvestres produzidos por Apis mellifera no Estado de São Paulo. In: Congresso Brasileiro de Apicultura, 11, 1996, Teresina. Resumos... Teresina: CBA, 1996b. p.344. 
Marchini, L.C.; Moreti, A.C.C.C. Características físico-químicas de amostras de mel de cinco diferentes espécies de eucaliptos. In: Simpósio Latino Americano de Ciência de Alimentos, 4, Campinas, 2001. Resumos... Campinas: SBCTA, 2001.p.42.

Noronha, P.R.G. Caracterização de méis cearenses produzidos por abelhas africanizadas: parâmetros químicos, composição botânica e colorimetria. Fortaleza: UFC, 1997. 147p. Dissertação Mestrado.

Ramalho, M. Valores e critérios do concurso de méis. Apicultura no Brasil, São Paulo, v.3, n.17, p.25-27, 1985.

Ramalho, M; Amaral, A.D. do; Azoubel, M.L. Mel: origem, caracterização e controle de qualidade. Apicultura no Brasil, São Paulo, v.4, n.23, p.25-36, 1987.

Rodrigues, A.G.L.R.; Marchini, L.C.; Haddad, M.L. Índice de diastase e HMF de mel extraído de colméias expostas diretamente ao sol e à sombra. In: Congresso Brasileiro de Apicultura, 11, Teresina, 1996. Resumos... Teresina: CBA, 1996.p.342.
Root, A.I. ABC y xyz de la apicultura: encyclopedia de la cria cientifica y prática de las abejas. Buenos Aires: Editorial Hemisfério Sur, 1985. 723p.

Silva, F. de A.S. The ASSISTAT software: statistical assistence. In: International Conference on Computers in Agriculture, 6, 1996, Cancun. Resumos.... Cancun: American Society of Agricultural Engineers, 1996. p.294-298.

Sodré, G.S.; Marchini, L.C.; Carvalho, A.L. Açúcares totais, redutores e sacarose de amostras de Apis mellifera (Hymenoptera: apidae) provenientes da região litoral norte no estado da Bahia. In: Simpósio Latino Americano de Ciência de Alimentos, 4, Campinas. Resumos... Campinas: SBCTA, 2001.p.114.

Vilhena, F.; Almeida-Muradian, L.B. Manual de análises físicoquímicas do mel. 1.ed. São Paulo: APACAME, 1999a. 16p.

Vilhena, F.; Almeida-Muradian, L.B. Análises-físico-químicas de méis de São Paulo. Mensagem Doce, São Paulo, n.53, p.17-19, 1999b.

White Júnior, J.W. La miel. In: Dadant, H. La colmena y la abeja melifera. Montevideo: Hemisfério Sul, 1989. cap.1, p.21-35. 